\title{
Integrating Community Health Workers Into Medical Homes
}

Elizabeth A. Rogers, MD, MAS

Sarab Turcotte Manser, $M A^{1}$

Joan Cleary, $M M^{2}$

Anne M. Joseph, MD, MPH

Eileen M. Harwood, $P b D^{3}$

Katbleen T. Call, $P b D^{4}$

'Division of General Internal Medicine, University of Minnesota Medical School, Minneapolis, Minnesota

${ }^{2}$ Minnesota Community Health Worker Alliance, St Paul, Minnesota

${ }^{3}$ Division of Epidemiology \& Community Health, University of Minnesota School of Public Health, Minneapolis, Minnesota

${ }^{4}$ Division of Health Policy \& Management University of Minnesota School of Public Health, Minneapolis, Minnesota

Conflicts of interest: authors report none.

\section{CORRESPONDING AUTHOR}

Elizabeth A. Rogers, MD, MAS Division of General Internal Medicine UMN Medical School 717 Delaware St SE, Suite 166 Minneapolis, MN 55414 earogers@umn.edu

\begin{abstract}
PURPOSE Though evidence supports the value of community health workers (CHWs) in chronic disease self-management support, and authorities have called for expanding their roles within patient-centered medical homes (PCMHs), few PCMHs in Minnesota have incorporated these health workers into their care teams. We undertook a qualitative study to (1) identify facilitators and barriers to utilizing a CHW model among PCMHs in Minnesota, and (2) define roles played by this workforce within the PCMH team.
\end{abstract}

METHODS We conducted 51 semistructured, key-informant interviews of clinic leaders, clinicians, care coordinators, CHWs, and staff from 9 clinics (5 with community health workers, 4 without). Qualitative analysis consisted of thematic coding aligned with interview topics.

RESULTS Four key conceptual themes emerged as facilitators and barriers to utilizing a CHW model: the presence of leaders with knowledge of CHW/s who championed the model, a clinic culture that favored piloting innovation vs maintaining established care models, clinic prioritization of patients' nonmedical needs, and leadership perceptions of sustainability. These health care workers performed common and clinic-specific roles that included outreach, health education and coaching, community resource linkage, system navigation, and facilitating communication between clinician and patient.

CONCLUSIONS We identified facilitators and barriers to adopting CHW roles as part of PCMH care teams in Minnesota and documented their roles being played in these settings. Our findings can be used when considering strategies to enhance utilization and integration of this emerging workforce.

Ann Fam Med 2018;16:14-20. https://doi.org/10.1370/afm.2171.

\section{INTRODUCTION}

ommunity health workers (CHWs), trusted frontline health workers who have a close understanding of the community served ${ }^{1}$ can be valuable contributors to the team-based and patient-centered care promoted through the patient-centered medical home (PCMH) model. ${ }^{2}$ $\mathrm{CHWs}$ complement roles played by traditional health professionals through culturally sensitive outreach, patient education, resource identification, case management, care coordination, and patient support. ${ }^{3.6}$ Interventions by $\mathrm{CHWs}$ supporting chronic disease self-management and preventive services show improved health care utilization, knowledge, self-care, adherence, health outcomes, and quality of life, particularly when these workers are integrated into primary care teams. ${ }^{4,-20}$ Their interventions are most often directed to and studied in underserved communities (racial and ethnic minority and low-income populations, federally qualified health care $[\mathrm{FQHC}]$ settings), where the integration of these workers show benefit. ${ }^{3,4,12,21-23}$ Health authorities have called for expanding interventions by $\mathrm{CHWs}$ among clinics serving these populations, and recent policies create a platform for greater community health worker integration..$^{24-26}$

Researchers have explored the experience of CHWs with integration into care teams and examined how health care delivery systems might be 
designed to effectively embed them. ${ }^{27-31}$ Experts have outlined implementation barriers (fragmented and disease-specific interventions, lack of clear work protocols, high turnover, variable performance, and a history of low-quality evidence), but argue that these barriers can be overcome through established standards to ensure a skilled CHW workforce, clearly defined roles, concrete implementation strategies, and an expanded scope of reimbursable services to include their services. ${ }^{5,6,25,26,32}$ Little research exists on whether these strategies are sufficient for wide adoption of CHWs within primary care and how clinics make decisions about this adoption.

Minnesota was the first state to create and offer a statewide competency-based CHW curriculum situated in postsecondary education. 19,33 Since 2009 Minnesota Medicaid has reimbursed diagnosis-related CHW patient education and self-management services. ${ }^{34}$ Even so, anecdotal information suggests that less than $10 \%$ of PCMHs in Minnesota have incorporated $\mathrm{CHWs}$ into their care teams. The transformation process required of primary care clinics when becoming PCMH-certified offers a unique view into clinic decision making regarding care team composition and structure. Our study aimed to identify facilitators and barriers to choosing to incorporate $\mathrm{CHWs}$ into the clinic team through the PCMH certification process. We also identify and describe roles being played by these health workers on PCMH teams.

\section{METHODS}

\section{Study Design}

To take advantage of the watershed moment of a new PCMH certification policy in Minnesota, we designed an exploratory qualitative study with key informants ${ }^{35}$ to understand their experience, perspectives, and observations of creating their new PCMH care team roles and structure.

\section{Setting}

Minnesota is a leader in PCMH implementation, with legislation establishing PCMH standards and clinic certification beginning in $2010 .{ }^{36}$ State PCMH certification is a redesign of care delivery and payment reform. ${ }^{36}$ State guidelines require that each clinic provide care coordination, but they do not stipulate what disciplines serve in this role. Within 4 years of initiating certification, 309 primary care clinics (41\%) were state-certified as PCMHs, largely located in the Minneapolis-St Paul metropolitan area. ${ }^{37}$

\section{Sample and Sample Selection}

We purposively sampled 10 PCMHs that (1) were certified for at least 1 year to gather perspectives from estab- lished, stable teams, and (2) represented clinics that had incorporated CHWs and those that had not. To examine facilitators to $\mathrm{CHW}$ integration, we invited 1 clinic from each health system known to have integrated these health workers into their teams (CHW clinics). To examine barriers to $\mathrm{CHW}$ integration, we invited clinics known to serve low-income patients of racial and ethnic minority (the populations for which $\mathrm{CHW}$ interventions have shown to be effective) but who had not integrated the health workers into their teams (non-CHW clinics). Clinics were the unit of analysis, with clinic staff as key informants speaking on behalf of the clinic.

Participating clinic leaders identified informants, who were invited to participate, representing the following: those leaders involved in PCMH certification decision making ( 1 to 2 per clinic) and frontline care team members ( 3 to 4 per clinic, including clinicians, care coordinators, $\mathrm{CHWs}$, and other staff).

\section{Measures and Measurement}

Our semistructured interview guide (see Supplemntal Appendix at http://www.annfammed.org/ content/16/1/14/suppl/DC1), designed for interviews lasting 45 to 60 minutes, included questions about decisions regarding PCMH team composition and structure, the use or potential use of CHWs, and the impact of PCMH certification on team structure, function, and member roles. This study focused on the narrative related to $\mathrm{CHW}$ adoption and roles. In addition, 1 leader per clinic completed a brief questionnaire describing clinic characteristics: clinic location, patient population characteristics, and staffing.

\section{Data Collection}

The investigatory team consisted of an internal medicine and pediatrics physician (E.R.) and a masters-leveltrained researcher (S.T.M.), both with previous experience in qualitative research. Both conducted in-person, semistructured, in-depth individual interviews that were audio-recorded in private offices at clinic sites in 2015. Interviewers made written field notes containing initial analytic impressions. ${ }^{38}$ Each successive interview was informed by issues heard in previous interviews and validated by prompting subsequent interviewees to address those issues. All study procedures were approved by the University of Minnesota Institutional Review Board.

\section{Data Analysis}

Interview audio recordings were transcribed, edited to ensure accuracy, and stripped of identifying information. Using NVivo 10 (QSR International 2014), we adopted an iterative approach to analysis based on thematic coding aligned with interview topics. We (E.R. and S.T.M.) used an a priori, deductive framework 
adapted from existing literature ${ }^{39}$ to review and code 3 transcripts independently. We then compared and discussed codes to develop agreement before moving to inductive coding and creation of the codebook to code the remaining transcripts. Through weekly analytic meetings, construct-level parent codes and their child dimensional subcodes were further refined using an iterative constant comparative method. ${ }^{40}$ Ongoing discussions, reflexive practices, and resulting memos helped identify emblematic themes, unifying concepts, and potential associations until thematic saturation was reached. Interview participants and community members who were not interviewed were asked to verify our interpretations as sound (member checking).

\section{RESULTS}

We interviewed 51 informants ( 45 women, 6 men) at 9 clinics agreeing to participate (1 delcined because of inadequate time), 5 with CHWs (Table 1). Mean interview duration was 48 minutes (range $=10$ - 68 minutes). Clinics represented mostly urban areas, with 1 clinic each in a small city and rural area, populations served varied in diversity.

We identified 4 themes related to both facilitators and barriers to clinic adoption of CHWs: leaders with knowledge of CHWs who championed the model, clinic culture that favored piloting innovation vs choosing familiar models, clinic prioritization of patients' nonmedical needs, and how leadership perceived sustainability. We describe these themes and offer verbatim and summarized examples of informants' perspectives from which these themes emerged. We also describe reported $\mathrm{CHW}$ roles on care teams.

\section{Facilitators to CHW Integration}

CHW Champions: Positive Perception of $\mathrm{CHW}$ Value In all $\mathrm{CHW}$ clinics, we noted clinician and administrative leaders championing the work of creating the new team model inclusive of CHWs. These decision makers expressed detailed knowledge of CHWs and had previous positive direct experiences with them. As 1 clinician respondent described, "I know about the community health worker [role]... and so I had the vision. I have been advocating for community health workers... for a long time."

Front-line staff cited the influence of champions as the most important factor for success:

Dr. [Name] was our super-motivated driving force.... It literally took her continually cramming it down people's throats...this is a new way of thinking, this is a new way of working with our patients (care coordinator).

Informants shared how champions furthered the positive image of CHWs within care teams: "I think there have been key champions within the care teams that

Table 1. Clinic Sample Characteristics

\begin{tabular}{|c|c|c|c|c|c|c|c|c|}
\hline Clinic & Location & Affiliation & $\begin{array}{l}\mathrm{MCa}^{\mathrm{a}} \\
\text { No. }\end{array}$ & Specialty & $\begin{array}{c}\text { Nonwhite } \\
\text { Race, \% }\end{array}$ & $\begin{array}{l}\text { Non-English } \\
\text { Language, \% }\end{array}$ & $\begin{array}{l}\text { Medicaid }{ }^{\mathrm{b}} \text { I } \\
\text { Medicare, \% }\end{array}$ & Interviews \\
\hline \multicolumn{9}{|c|}{ CHW clinics } \\
\hline A & Urban & Health system & 37 & $\begin{array}{l}\text { Internal } \\
\text { medicine }\end{array}$ & 15 & 11 & $7 / 45$ & $\begin{array}{l}\text { 5: CC, CHW, MC, } \\
\text { Sup (2) }\end{array}$ \\
\hline B & Small city & Health system & 21 & Pediatric & 17 & 9 & $27 / 0.1$ & $\begin{array}{l}\text { 7: BH, CC, CHW, } \\
\text { MC (2), PL, Sup }\end{array}$ \\
\hline $\mathrm{C}$ & Rural & Health system & 10 & Family medicine & NA & 1 & $17 / 45$ & $\begin{array}{l}\text { 5: CC, CHW, CM, } \\
\text { MA, MC }\end{array}$ \\
\hline $\mathrm{D}$ & Urban & Health system & 23 & $\begin{array}{l}\text { Internal } \\
\text { medicine }\end{array}$ & 65 & 17 & $55 / 23$ & $\begin{array}{l}\text { 5: CC, CHW, CM, } \\
\text { MC (2) }\end{array}$ \\
\hline$E$ & Urban & Health system & 9 & Family medicine & 54 & 36 & $44 / 12$ & $\begin{array}{l}\text { 5: CHW (2), CM, } \\
\text { MC, PL }\end{array}$ \\
\hline \multicolumn{9}{|c|}{ Non-CHW clinics } \\
\hline $\mathrm{G}$ & Urban & Health system & 19 & Family medicine & 89 & 8 & $80 / 5$ & $\begin{array}{l}\text { 6: CC, CM, MC, Ph, } \\
\text { PL, Sup }\end{array}$ \\
\hline $\mathrm{H}$ & Urban & $\mathrm{FQHC}$ & 9 & Family medicine & 39 & 58 & $41 / 12$ & 5: CC (2), CM, PL, Sup \\
\hline 1 & Urban & $\mathrm{FQHC}$ & 15 & Primary care & 80 & 43 & $58 / 8$ & $\begin{array}{l}\text { 5: CC, CM, MA, PL, } \\
\text { Sup }\end{array}$ \\
\hline
\end{tabular}

$\mathrm{BH}=$ behavioral health worker; $\mathrm{CC}=$ care coordinator; $\mathrm{CHW}=$ community health worker; $\mathrm{CM}=$ clinic manager; $\mathrm{FQHC}=\mathrm{federally}$ qualified health center; $\mathrm{MA}=$ medical assistant or interpreter; $\mathrm{MC}=$ medical clinician; $\mathrm{NA}=$ data not available; $\mathrm{Ph}=$ clinical pharmacist; $\mathrm{PL}=$ physician leader or champion, or medical director; Sup = supervisor of care coordinators, CHWs, or nursing; SW = social worker.

${ }^{a} \mathrm{MC}=$ includes physicians, nurse practitioners, and physician assistants.

${ }^{\mathrm{b}}$ Medicaid $=$ includes other government and medical assistance funds.

cPrimary care $=$ family medicine, internal medicine, and pediatrics. 
helped identify the benefits of the CHWs" (clinician), and across the health care system: "People who were passionate about their cause and did the presentations through administration, showed the value...[and] the evidence" (care coordinator supervisor).

\section{Clinic Culture: Embracing and Piloting Innovation} All clinic leaders described the influence of care team redesign mandates required for PCMH certification. CHW clinic leaders viewed these certification mandates as an opportunity for workforce innovation. Respondents were descriptive of their innovative culture: "We [are] constantly doing research on... how will [patients] really benefit? We're constantly trying to improve" (care coordinator).

$\mathrm{CHW}$ clinics secured grant funding to partially support an initial CHW pilot program. "We got money from [funder]...I think that was what made the decision to hire CHWs" (clinic manager). Some "started small" to work out "the kinks" in implementation. A CHW described the integration process as stepwise, at times with skeptical team members: "So I think at first...nobody knew [eg], why is she even a clinic employee? [Some] care team members seemed to get it before the clinic doctors did." Clinic staff directly observed benefits, supporting notable successes. "I honestly think [CHW integration] has been all benefit" (medical director), and establishing "credibility and trust from the board and the clinic" (care coordinator supervisor). These pilots provided data to decision makers to support the CHW role in on-going clinic operations. One physician leader described expansion as "widespread acknowledgement that [CHW integration] is a good thing...that is spreading within [our health system]."

\section{Acknowledging Patients' Nonmedical Needs}

In $\mathrm{CHW}$ clinics, informants described the importance of the psychosocial, cultural, and socioeconomic needs of their patient population in managing health.

The fact that the patient doesn't have heat, or they don't know how to get food.... These are the patients that need help beyond what medicine can give them...that frustrated us the most because we feel like we can't do anything. And now [with CHWs] it's a different story (clinic administrator).

Addressing cultural needs was also a driver for 1 physician leader working in a clinic caring for a specific minority ethnic population: "Our cultural knowledge was so limited."

\section{Positive Perception of Sustainability}

For CHW clinic leaders, the financial sustainability potential of $\mathrm{CHWs}$ was a motivating factor. One
PCMH director described the design of their team structure so that nonmedical tasks would not be "squeezed in" by a more traditional staffing model: "That there will be someone fully dedicated to [care coordination]...was a huge win.... The use of CHWs makes that a little bit more financially sustainable."

\section{Barriers to $\mathrm{CHW}$ Integration}

Lack of CHW Champions: Limited Awareness of $\mathrm{CHW}$ Value

Many non-CHW clinic leaders and staff had limited knowledge of $\mathrm{CHW}$ roles or no first-hand experience. "I've heard about $[\mathrm{CHWs}]$... but I haven't really heard a model where somebody said, 'That's been really effective'"' (nursing supervisor). These clinics did not have a leader championing the $\mathrm{CHW}$ model for consideration in care team redesign. One medical director described a single outside clinic's anecdotal experience of CHWs as an influential deterrent. Although that organization relayed that "outreach was great," disparate experience included " [CHWs] couldn't quite manage at the level that they wanted to," prompting this leader to adopt a constrained view of $\mathrm{CHW}$ potential.

\section{Clinic Culture: Choosing Familiar and \\ Established Models}

When considering how to meet PCMH requirements, most non-CHW clinic leaders built their teams with established health professions. One clinic manager shared that they drafted a care coordinator job description "so pretty much anybody could apply" and mapped how existing staff could be reassigned. Informants pointed to familiarity, as well as perceived versatility, when choosing staffing models. "Part of the reason I wanted to create RN [registered nurse] care coordinators is you need RNs for other jobs, too" (medical director). Some leaders shared that innovation could be difficult because of their system's centralized, top-down decision making.

When asked whether they considered incorporating $\mathrm{CHWs}$ into their clinic teams, informant responses reflected feelings of uncertainty- "We just haven't been able to figure out how to make it work" — and 1 clinic manager had concerns that $\mathrm{CHW}$ incorporation may be "risky." Given room to expand on their views, informants in managerial roles identified underlying perceptions about complexities in hiring a new role (it "sounds straight forward on the surface; but, it's pretty complicated"), assumed capabilities ("you don't want to throw people into situations that they're not able to handle"), and supervision (lack of resources for "adequate supervision" of CHWs). Concern about supervision capacity and structure emerged as a particularly important barrier. 


\section{Focus on Patients' Medical Needs}

In non-CHW clinics, informants focused on patients' medical needs. One clinic decided that registered nurses would fill the care coordinator role and addressed patient language needs by using bilingual nurses. A care coordinator supervisor expressed little understanding of $\mathrm{CHW}$ competency with diagnosisrelated patient education: "I worry if somebody is explaining something wrong.... One of [the CHWs from a conference] was on diabetes, and right away I was, like, 'How much do you know about diabetes?'" One medical director could not envision what role a $\mathrm{CHW}$ might play for patients with complex medical issues, demonstrating a medicalized view of complexity: "When I already have a diabetic whose [hemoglobin] $A_{1 C}$ is $12 \%$ or their blood pressure is $160[\mathrm{mmHg}$, then it's a little bit harder for me to imagine how [the CHW] would fit into that team."

\section{Perceived Uncertainty of Sustainability}

We heard leader concerns about financing CHWs. One operations director said, "Yes...I haven't figured out how to fund [CHWs].... That's the only road block. Our clinics are not profitable, and you can only go so much in the red." Clinic leaders were also committed to sustaining hired staff, which led to hesitation about hiring CHWs. "I figured that I was always going to have RNs, I wouldn't always have CHWs. Even though they're cheaper, they are also easier to let go if they only have a single function" (medical director).

\section{Team Structure and CHW Roles}

No team structure was alike among participating clinics. For example, care coordinators ranged from $\mathrm{CHWs}$ to certified medical assistants to social workers to registered nurses. There was particular variability among the $5 \mathrm{CHW}$ clinics, though all $\mathrm{CHWs}$ held certificates. This heterogeneity provided insights into how teams functioned and which roles were played. Table 2 outlines $\mathrm{CHW}$ roles, using categories guided by previous literature. ${ }^{41-45}$ Several patient-facing roles were com-

\section{Table 2. Community Health Worker Roles Within the PCMH Team}

\section{Common roles}

Outreach, health education, community resource linkage, health coaching, social support, system navigation, facilitation of communication between patient and clinician

Unique, clinic-specific roles

Care coordination, home-based support, community health promotion, language-specific care coordination, cultural liaison, acting as "eyes and ears" in the community for clinic team

$\mathrm{PCMH}=$ patient-centered medical home mon: outreach and navigation to increase access, education and coaching, social support, facilitating communication with the health care system, and linkage to referrals and community resources.

Clinic-specific roles appeared to depend upon perception of patient need and the extent to which CHWs functioned as an extension into the community. For example, culture-specific needs influenced the uptake of CHWs in 2 clinics and defined their roles and activities. One medical director said that after their $\mathrm{CHW}$ was hired, "our eyes were opened to what the communication, access, and transportation barriers were" for 1 specific ethnic group. Three of the $6 \mathrm{CHW}$ respondents spoke a non-English language critical to their role. In 2 other clinics, community resource connections and home visits were recognized as important patient needs; CHWs served as the "eyes and ears" (medical director) of the clinic team in the community.

\section{DISCUSSION}

Findings from Minnesota clinics serving low-income populations that underwent $\mathrm{PCMH}$ transformation suggest specific facilitators and barriers to adopting a CHW team member. Integration requires a champion with awareness of potential CHW value, a clinic culture of innovation with pilot resources, perception of patient needs spanning medical and nonmedical determinants of health, and finding sustainable reimbursement strategies. Many barriers still exist to $\mathrm{CHW}$ adoption, the most foundational being lack of awareness by leadership of the value of the $\mathrm{CHW}$ role.

Non-CHW clinic informants reported difficulty imagining financial sustainability within the current reimbursement system, reinforcing findings from others who describe reimbursement as one of the most important policy problems affecting the emergence of the CHW workforce. ${ }^{5,25,26,32}$ A sole focus on reimbursement, however, misses other identified obstacles to embracing workforce innovation, including concerns about the risk of hiring a new workforce member, supervision, and a medicalized view of patients.

Previous studies share lessons learned from integrating CHWs into a team-based practice, ${ }^{27-31}$ but only 1 included descriptions of the preimplementation decision making that led to piloting the $\mathrm{CHW}$ model. Their results echoed themes from our study, including having an awareness of the CHW model and influential champions, and building institutional support through documented return on investment. ${ }^{29}$ Our study is unique in its focus on decision making concerning integration of $\mathrm{CHWs}$, highlighting barriers and additional facilitators, including embracing organizational innovation, securing resources for a pilot program, and valuing patients' 
nonmedical needs. Piloting the CHW model built a sense of trust and institutional support.

An appreciation for these health workers' value was critical and yet was missing in most of non-CHW clinic informants. This finding likely reflects the relative dearth of mainstream discussion about CHWs in the United States until the past half-decade. ${ }^{25}$ Although mounting evidence of CHW effectiveness should allay some concerns, more work is needed.

In participating $\mathrm{PCMHs}$, care team structure and composition were heterogeneous, and CHWs played a variety of common and clinic-specific roles. Consistent with recent documentation within 5 FQHCs ${ }_{1}^{42,46}$ $\mathrm{CHW}$ roles spanned patient self-management support, navigation of health and social systems, patient education, case management, outreach, individual care plans, social support, and contributions to behavioral and mental health. This variety of $\mathrm{CHW}$ roles may require new strategies (eg, interprofessional education) to demonstrate the value and potential of this new workforce to traditional health care professionals.

Our study has some limitations. The sample of certified PCMHs does not, by design, represent all primary care clinics. Our non-CHW clinics focused on urban safety-net clinics because their patient populations reflect those in studies showing CHW effectiveness; we may have missed rural barriers to $\mathrm{CHW}$ adoption. Biases may exist among clinics that sought certification earlier than others; $\mathrm{PCMHs}$ in our study were among the first $40 \%$ in the state to pursue certification. ${ }^{37}$ The direction of these potential biases is not clear, however. Finally, the data analysis team attempted to minimize personal biases by acknowledging them, using consensus, and inviting stakeholder review of early findings to ensure the quality of interpretations.

Future research should aim to understand effective $\mathrm{CHW}$ supervision capacity within a PCMH model and to identify which team structures and $\mathrm{CHW}$ roles are linked to improved team performance and patient outcomes, particularly with regard to health equity. The PCMH's focus on team-based multidisciplinary care provides an opportunity for the CHW workforce and for continued policy development, particularly as the US health care system focuses on decreasing costs. As more practices seek PCMH certification, facilitators and barriers will influence how clinics choose care team members and structure, particularly whether they embrace the integration of emerging workforce members such as CHWs.

To read and comment in response to this article, see it online at http://www.AnnFamMed.org/content/16/1/14.

Key words: medical home; community health workers; primary care; health workforce
Submitted January 12, 2017; submitted, revised June 28, 2017; accepted July 21, 2017.

Acknowledgements: The authors thank the clinic leadership and staff who generously gave of their time to participate in the interviews and share invaluable perspectives.

Funding support: Research reported in this publication was supported by the National Center for Advancing Translational Sciences of the National Institutes of Health Award Number UL1TR000114.

Disclaimer: The content is solely the responsibility of the authors and does not necessarily represent the official views of the National Institutes of Health.

Previous presentations: Preliminary results from this work have been presented at the American Public Health Association Annual Meeting, November 3, 2015, and at the Society for General Internal Medicine Annual Meeting, May 12, 2016.

Supplementary materials: Available at http://www.AnnFamMed. org/content/16/1/14/suppl/DC1/.

\section{References}

1. American Public Health Association. Community health workers. https://www.apha.org/apha-communities/member-sections/ community-health-workers. Updated 2016. Accessed Aug 23, 2016.

2. Rosenthal TC. The medical home: growing evidence to support a new approach to primary care. J Am Board Fam Med. 2008;21(5): 427-440.

3. Norris SL, Chowdhury FM, Van Le K, et al. Effectiveness of community health workers in the care of persons with diabetes. Diabet Med. 2006;23(5):544-556.

4. Otero-Sabogal R, Arretz D, Siebold S, et al. Physician-community health worker partnering to support diabetes self-management in primary care. Qual Prim Care. 2010;18(6):363-372.

5. Islam N, Nadkarni SK, Zahn D, Skillman M, Kwon SC, Trinh-Shevrin C. Integrating community health workers within Patient Protection and Affordable Care Act implementation. J Public Health Manag Pract. 2015;21(1):42-50.

6. Daaleman TP, Fisher EB. Enriching patient-centered medical homes through peer support. Ann Fam Med. 2015;13(Suppl 1):S73-S78.

7. Kim KB, Kim MT, Lee HB, Nguyen T, Bone LR, Levine D. Community health workers versus nurses as counselors or case managers in a self-help diabetes management program. Am J Public Health. 2016; 106(6):1052-1058.

8. Palmas W, Findley SE, Mejia M, et al. Results of the northern Manhattan diabetes community outreach project: a randomized trial studying a community health worker intervention to improve diabetes care in Hispanic adults. Diabetes Care. 2014;37(4):963-969.

9. Krieger J, Song L, Philby M. Community health worker home visits for adults with uncontrolled asthma: the HomeBASE Trial randomized clinical trial. JAMA Intern Med. 2015;175(1):109-117.

10. Brownstein JN, Chowdhury FM, Norris SL, et al. Effectiveness of community health workers in the care of people with hypertension. Am J Prev Med. 2007;32(5):435-447.

11. Philis-Tsimikas A, Fortmann A, Lleva-Ocana L, Walker C, Gallo LC. Peer-led diabetes education programs in high-risk Mexican Americans improve glycemic control compared with standard approaches: a Project Dulce promotora randomized trial. Diabetes Care. 2011; 34(9):1926-1931.

12. Balcazar H, Alvarado M, Ortiz G. Salud Para Su Corazon (health for your heart) community health worker model: community and clinical approaches for addressing cardiovascular disease risk reduction in Hispanics/Latinos. J Ambul Care Manage. 2011;34(4):362-372. 
13. Carrasquillo O, Lebron C, Alonzo Y, Li H, Chang A, Kenya S. Effect of a community health worker intervention among latinos with poorly controlled type 2 diabetes: The miami healthy heart initiative randomized clinical trial. JAMA Intern Med. 2017;177(7): 948-954.

14. Fisher EB, Boothroyd RI, Coufal MM, et al. Peer support for selfmanagement of diabetes improved outcomes in international settings. Health Aff (Millwood). 2012;31(1):130-139.

15. Pati S, Ladowski KL, Wong AT, Huang J, Yang J. An enriched medical home intervention using community health workers improves adherence to immunization schedules. Vaccine. 2015;33(46): 6257-6263.

16. Thompson JR, Horton C, Flores C. Advancing diabetes selfmanagement in the Mexican American population: a community health worker model in a primary care setting. Diabetes Educ. 2007; 33(Suppl 6):159S-165S.

17. Viswanathan M, Kraschnewski JL, Nishikawa B, et al. Outcomes and costs of community health worker interventions: a systematic review. Med Care. 2010;48(9):792-808.

18. Foster G, Taylor SJ, Eldridge SE, Ramsay J, Griffiths CJ. Selfmanagement education programmes by lay leaders for people with chronic conditions. Cochrane Database Syst Rev. 2007;(4)(4): CD005108.

19. The Institute for Clinical and Economic Review. Community Health Workers: A Review of Program Evolution, Evidence on Effectiveness and Value, and Status of Workforce Development in New England. Draft report. http://icer-review.org/wp-content/uploads/2011/04/ CHW-Draft-Report-05-24-13-MASTER1.pdf. Published May 24, 2013.

20. Rosenthal EL, Wiggins N, Ingram M, Mayfield-Johnson S, De Zapien JG. Community health workers then and now: an overview of national studies aimed at defining the field. J Ambul Care Manage. 2011;34(3):247-259.

21. Viswanathan M, Kraschnewski J, Nishikawa B, et al. Outcomes of community health worker interventions. Evid Rep Technol Assess (Full Rep). 2009;(181)(181):1-144, A1-2, B1-14, passim.

22. Tang TS, Funnell $M$, Sinco $B$, et al. Comparative effectiveness of peer leaders and community health workers in diabetes selfmanagement support: results of a randomized controlled trial. Diabetes Care. 2014;37(6):1525-1534.

23. Adair R, Wholey DR, Christianson J, White KM, Britt H, Lee S. Improving chronic disease care by adding laypersons to the primary care team: a parallel randomized trial. Ann Intern Med. 2013;159(3): 176-184.

24. Centers for Disease Control and Prevention. Division for Heart Disease and Stroke Prevention. A policy Brief on Commnity Health Workers. Addressing chronic disease through community health workers: A policy and systems-level approach. $2^{\text {nd }} \mathrm{ed}$. https://www. cdc.gov/dhdsp/docs/chw_brief.pdf. Published Apr 2015.

25. Kangovi S, Grande D, Trinh-Shevrin C. From rhetoric to realitycommunity health workers in post-reform U.S. health care. N Engl J Med. 2015;372(24):2277-2279.

26. Hynes DM, Buscemi J, Quintiliani LM; Society of Behavioral Medicine Health Policy Committee. Society of Behavioral Medicine (SBM) position statement: SBM supports increased efforts to integrate community health workers into the patient-centered medical home. Transl Behav Med. 2015;5(4):483-485.

27. Collinsworth A, Vulimiri M, Snead C, Walton J. Community health workers in primary care practice: redesigning health care delivery systems to extend and improve diabetes care in underserved populations. Health Promot Pract. 2014;15(2)(Suppl):51S-61S.

28. Wennerstrom A, Bui T, Harden-Barrios J, Price-Haywood EG. Integrating community health workers into a patient-centered medical home to support disease self-management among Vietnamese Americans: lessons learned. Health Promot Pract. 2015;16(1):72-83.

29. Findley S, Matos S, Hicks A, Chang J, Reich D. Community health worker integration into the health care team accomplishes the triple aim in a patient-centered medical home: a Bronx tale. J Ambul Care Manage. 2014;37(1):82-91.
30. Allen CG, Escoffery C, Satsangi A, Brownstein JN. Strategies to improve the integration of community health workers into health care teams: "A little fish in a big pond." Prev Chronic Dis. 2015;12: E154.

31. Johnson SL, Gunn VL. Community health workers as a component of the health care team. Pediatr Clin North Am. 2015;62(5): 1313-1328.

32. Martinez J, Ro M, Villa NW, Powell W, Knickman JR. Transforming the delivery of care in the post-health reform era: what role will community health workers play? Am J Public Health. 2011;101(12): e1-e5.

33. Agency for Healthcare Research and Quality. Alliance creates community health workers' scope of practice, training curriculum, certificate program, and reimbursement strategy, expanding their integration into the health system to reduce health disparities. https://innovations.ahrq.gov/profiles/alliance-creates-communityhealth-workers-scope-practice-training-curriculum-certificate. Published 2012.

34. Minnesota statute 256B.0625, subdivision 49. 2009:Sec. 4. Minnesota Statutes 2009 Supplement.

35. Corbin JM. Basics of Qualitative Research: Techniques and Procedures for Developing Grounded Theory. 3rd ed. Los Angeles, CA: Sage Publications, Inc; 2008.

36. Minnesota Department of Health. Health Care Homes: Background Information \& Reports. http://www. health.state.mn.us/healthreform/ homes/about/. Updated 2016. Accessed Aug 23, 2016.

37. Wholey DR, Finch M, Shippee ND, et al. Evaluation of the state of Minnesota's health care homes initiative: Evaluation reports for years 2010-2014. http://www.health.state.mn.us/healthreform/ homes/legreport/docs/hch2016report.pdf. Published Dec 2015.

38. Creswell JW. Research Design: Qualitative, Quantitative, and Mixed Methods Approaches. 4th ed. Thousand Oaks, CA: Sage Publications; 2014.

39. Bradley EH, Curry LA, Devers KJ. Qualitative data analysis for health services research: developing taxonomy, themes, and theory. Health Serv Res. 2007;42(4):1758-1772.

40. Glaser BG. The Discovery of Grounded Theory; Strategies for Qualitative Research. Chicago, IL: Aldine Pub Co; 1967.

41. Collinsworth AW, Vulimiri M, Schmidt KL, Snead CA. Effectiveness of a community health worker-led diabetes self-management education program and implications for $\mathrm{CHW}$ involvement in care coordination strategies. Diabetes Educ. 2013;39(6):792-799.

42. Reinschmidt KM, Ingram M, Morales S, et al. Documenting community health worker roles in primary care: Contributions to evidence-based integration into health care teams, 2015. J Ambul Care Manage. 2017;40(4):305-315.

43. Rosenthal EL, Wiggins N, Brownstein JN, Johnson S, Borbon IA, De Zapien JG. The Final Report of the National Community Health Advisor Study: Weaving the Future. http://crh.arizona.edu/sites/ default/files/pdf/publications/CAHsummaryALL.pdf. Published Jun 1998.

44. Rosenthal EL, Rush CH, Allen CG. Understanding scope and competencies: A contemporary look at the united states community health worker field - progress report of the community health worker core consensus (C3) project: Building national consensus on CHW core roles, skills, and qualities. 2016. http://c3report.chwsurvey.com. Accessed Sep 2, 2016.

45. Matos S, Findley S, Hicks A, Legendre Y, Do Canto L. Paving a path to advance the community health worker workforce in new york state: A new summary report and recommendations. http:// nyshealthfoundation.org/resources-and-reports/resource/paving-apath-to-advance-the-community-health-worker-workforce-in-newyork. Published Oct 2011. Accessed Oct 3, 2016.

46. Volkmann K, Castañares T. Clinical community health workers: linchpin of the medical home. J Ambul Care Manage. 2011;34(3): 221-233. 